Психология. Журнал Высшей школы экономики.

2021. T. 18. № 3. C. 506-524. DOI: 10.17323/1813-8918-2021-3-506-524

\title{
РОЛЬ ВОВЛЕЧЕННОСТИ ПРИ ВОЗНИКНОВЕНИИ АССИМИЛЯЦИИ/КОНТРАСТА В ОЦЕНКАХ ПОТРЕБИТЕЛЬСКОЙ УДОВЛЕТВОРЕННОСТИ
}

\begin{abstract}
Б.Г. РЕБЗУЕВ
${ }^{a}$ Федеральное государственное бюджетное образовательное учреждение высшего образования «Российский государственный педагогический университет им. А.И. Герцена», 191186, Санкт-Петербург, набережная реки Мойки, 48
\end{abstract}

\begin{abstract}
Резюме
Исследования показывают, что одной из ключевых детерминант потребительской удовлетворенности является неподтверждение ожиданий, под которым понимаются воспринимаемые различия между функционированием продукта и ожиданиями, сформированными перед его покупкой. При этом позитивное неподтверждение (когда функционирование превосходит ожидания) приводит к высокой, а негативное (когда оно уступает ожиданиям) к низкой удовлетворенности. В настоящем исследовании изучалась роль вовлеченности при возникновении эффектов ассимиляции (снижения оценок неподтверждения и смещения оценок удовлетворенности в сторону ожиданий) и эффектов контраста (повышения оценок неподтверждения и смещения оценок удовлетворенности в противоположную сторону), а также процессы, приводящие к эффектам контраста. И формулировались две гипотезы о том, что вовлеченность будет вызывать эффекты контраста и что такие эффекты будут возникать в результате активизации когнитивных процессов, приводящих к усилению воспринимаемых различий между функционированием продукта и ожиданиями. Гипотезы проверялись в лабораторном эксперименте, манипулировавшем функционированием продукта (качеством спрея для экранов мониторов), ожиданиями и уровнями вовлеченности участников и позволявшем изучить два вида эффектов ассимиляции/контраста, негативных и позитивных, возникающих в результате завышенных и заниженных ожиданий относительно продукта. Эксперимент показал, что вовлеченность действительно вызывала эффекты негативного и позитивного контраста в оценках неподтверждения ожиданий и удовлетворенности, за одним исключением: она не вызывала позитивного контраста в оценках удовлетворенности. В статье рассматриваются возможные причины такого результата. Эксперимент также поддержал существование цепочки процессов, приводящих к эффектам контраста, в которой вовлеченность повышает уровень активации субъекта, активация стимулирует когнитивную активность, когнитивная активность повышает точность суждений в оценках продукта, а точность суждений усиливает воспринимаемые различия между функционированием продукта и ожиданиями. Обсуждаются ограничения, вопросы для будущих исследований и следствия для производителей и продавцов, вытекающие из эффектов ассимиляции/контраста.
\end{abstract}

Ключевые слова: вовлеченность, потребительская удовлетворенность, неподтверждение ожиданий, ожидания, функционирование продукта, эффект ассимиляции, эффект контраста, диапазон принятия, диапазон отвержения, теория социальных суждений, модель вероятности осмысления. 
Потребительская удовлетворенность (ПУ) - «реакция потребителя на достижение чего-либо желавшегося или ожидавшегося, степень, в которой уровень такого достижения оказывается приятным или неприятным» (Oliver, 2014, p. 23). Потребительская удовлетворенность вызывает интерес исследователей ввиду ее связи с повторными покупками (Curtis et al., 2011; Szymanski, Henard, 2001). Большинство исследований ПУ проводилось в русле «парадигмы неподтверждения ожиданий», согласно которой ключевой предпосылкой ПУ является неподтверждение ожиданий, возникающее в результате сравнения потребителем функционирования продукта с ожиданиями, сформированными перед его покупкой. При этом позитивное неподтверждение (когда продукт превышает ожидания) приводит к высокой ПУ, негативное непотверждение (когда продукт уступает ожиданиям) - к низкой ПУ, а подтверждение (когда он отвечает ожиданиям) к умеренной ПУ. Хотя эта парадигма получила эмпирическую поддержку (Oliver, 2014; Szymanski, Henard, 2001; Yi, 1990), в отдельных исследованиях наблюдались значительные расхождения во мнениях о связях ожиданий и воспринимаемого функционирования продукта с неподтверждением и ПУ, что побудило исследователей обратиться к изучению переменных, способных модерировать (усиливать или ослаблять) такие связи. При этом их наибольшее внимание привлекла вовлеченность, предположительно усиливающая неподтверждение и эффекты контраста в оценках ПУ (Altunel, Kocak, 2017; Babin et al., 1994; Oliver, Bearden, 1983; Richins, Bloch, 1991; Shaffer, Sherrell, 1997; Spreng, Sonmez, 2000; Tam, 2011). Однако поскольку такие исследования приводили к смешанным результатам, что может объясняться ограниченными возможностями контроля над посторонними переменными из-за их корреляционного характера, целью настоящего исследования являлась проверка модерирующей роли вовлеченности при возникновении эффектов ассимиляции/контраста в оценках неподтверждения и ПУ в контролируемых условиях лабораторного эксперимента. Другой его целью являлось изучение не рассматривавшихся в этих исследованиях процессов, благодаря которым вовлеченность выполняет свою модерирующую роль.

В психологии понятие вовлеченности (involvement) используется для объяснения формирования или изменения аттитюда под влиянием убеждающего сообщения и изучается в рамках трех исследовательских традиций: теории социального суждения (Sherif, Hovland, 1961), модели вероятности осмысления (Petty, Cacioppo, 1986; Petty, Briñol, 2012) и исследований конформизма (Zimbardo, 1960). Первая (Sherif, Hovland, 1961) ставит эффективность убеждающего сообщения в зависимость от того, попадает выражаемый в ней аттитюд в диапазон принятия (область аттитюдов, с которыми субъект склонен соглашаться) или отвержения (область аттитюдов, с которыми он не согласен). В первом случае он склонен оценивать такой аттитюд как более близкий к своей позиции, чем в действительности (эффект ассимиляции), а во втором как отличающийся от нее (эффект контраста), при этом высокая вовлеченность сужает диапазон принятия, вызывая эффекты контраста и усиливая несогласие субъекта с убеждающим сообщением. В таких исследованиях 
вовлеченностью манипулируют за счет привлечения участников со сложившейся устойчивой позицией по рассматриваемой важной проблеме (обычно в области семьи, религии или политики) и с ее отсутствием. В отличие от первой вторая традиция (Petty, Cacioppo, 1986; Petty, Briñol, 2012) оперирует ситуациями со сравнительно незнакомыми субъектам проблемами и ставит эффективность убеждающего сообщения в зависимость от качества аргументов, приводимых в поддержку отстаиваемой в нем позиции. В подобных ситуациях высокая вовлеченность стимулирует субъекта к активному осмыслению этих аргументов с последующим формированием у него благоприятного и устойчивого аттитюда. В таких исследованиях вовлеченностью манипулируют за счет важности/неважности для субъекта последствий принятия им позиции, отстаиваемой в убеждающем сообщении (например, в случае студентов - необходимости введения дополнительного выпускного квалификационного экзамена в университете, в случае потребителей - предложения после просмотра рекламы выбрать в подарок за участие в исследовании один из брендов продукта, который будет показываться в рекламе, или какого-то другого продукта). Третья традиция (Zimbardo, 1960) ставит эффективность убеждающего сообщения в зависимость от того, предупреждают (высокая вовлеченность) или не предупреждают (низкая вовлеченность) субъекта о том, что после предъявления сообщения ему предстоит отстаивать свою позицию по рассматриваемой в сообщении проблеме перед незнакомой аудиторией. С учетом различного характера манипуляций вовлеченностью в этих трех традициях и различных последствий убеждающих сообщений (отсутствие изменений в первоначальном аттитюде, формирование благоприятного аттитюда и формирование менее поляризованного аттитюда) Джонсон и Игли (Johnson, Eagly, 1989) предложили рассматривать три разных вида вовлеченности: вызываемую активизацией устойчивых ценностей (релевантную ценностям), активизацией способности достигать желательных последствий (релевантную последствиям) и активизацией публичного Я (релевантную самопрезентации). А под самой вовлеченностью понимать «мотивационное состояние, вызываемое ассоциацией между активированным аттитюдом и некоторым аспектом Я-концепции» (Ibid., p. 293).

Впоследствии представления о диапазонах принятия-отвержения, эффектах ассимиляции-контраста и видах вовлеченности, разработанные психологами, были заимствованы исследователями ПУ для объяснения влияния ожиданий и неподтверждения ожиданий на оценки продукта и удовлетворенности. При этом ожидания относительно продукта стали рассматриваться в виде континуума, состоящего из уровней функционирования ранее приобретавшихся вариантов такого продукта с центральной областью, представленной более частыми и привычными уровнями их функционирования (диапазоном принятия), и двумя крайними областями, представленными более редкими и непривычными уровнями - диапазоном отвержения (Woodruff et al., 1983). B данном контексте при небольших воспринимаемых расхождениях между продуктом и ожиданиями (при его попадании в диапазон принятия с привычными уровнями функционирования) прогнозируется эффект ассимиляции, приводящий к 
искажению оценок продукта в сторону ожиданий, тогда как при больших воспринимаемых расхождениях (при его попадании в диапазон отвержения) эффект контраста, приводящий к искажению оценок продукта в противоположную сторону (Olshavsky, Miller, 1972). В дополнение исследователи ПУ также выделили два вида вовлеченности, соответствующие по смыслу вовлеченности, релевантной ценностям и последствиям: устойчивую и ситуационную вовлеченность, отражающие постоянный уровень интереса к категории продукта, не зависящий от ситуации, и временное усиление такого интереса к категории продукта из-за специфических ситуационных влияний (Bloch, Richins, 1983; Houston, Rothschild, 1978).

Все эти соображения естественным образом наводили на следующую идею о модерирующей роли вовлеченности во влиянии ожиданий и функционирования на неподтверждение ожиданий и ПУ: высокая вовлеченность будет усиливать воспринимаемые различия между функционированием и ожиданиями и приводить к эффектам контраста в оценках ПУ (смещать их в противоположную сторону от ожиданий), тогда как низкая вовлеченность будет ослаблять такие различия и приводить к эффектам ассимиляции (смещать их в сторону ожиданий). Такая идея проверялась в ряде исследований (Altunel, Kocak, 2017; Babin et al., 1994; Oliver, Bearden, 1983; Richins, Bloch, 1991; Shaffer, Sherrell, 1997; Spreng, Sonmez, 2000; Tam, 2011), при этом из-за их корреляционного характера ее пришлось переформулировать из терминов эффектов различий в термины эффектов связей: при высокой вовлеченности ПУ будет сильнее подвержена влиянию неподтверждения, тогда как при низкой вовлеченности - влиянию предварительных ожиданий (Oliver, Bearden, 1983, с. 254). А чтобы учесть влияние вовлеченности на неподтверждение (т.е. на воспринимаемые различия между функционированием и ожиданиями) и последующие оценки ПУ, пришлось сформулировать дополнительную гипотезу: вовлеченность будет генерировать более крайние оценки неподтверждения и ПУ (Ibid.). Однако эти исследования привели к смешанным результатам. Так, при проверке первой гипотезы неподтверждение коррелировало сильнее с ПУ при высокой вовлеченности, чем при низкой в одних исследованиях (Babin et al., 1994; Shaffer, Sherrell, 1997; Spreng, Sonmez, 2000), но не в других (Oliver, Bearden, 1983; Richins, Bloch, 1991; Tam, 2011), а ожидания не коррелировали сильнее с ПУ при низкой, чем при высокой вовлеченности (Oliver, Bearden, 1983; Shaffer, Sherrell, 1997). Сходным образом при проверке второй гипотезы вовлеченность вызывала более высокие оценки неподтверждения и ПУ в одних исследованиях (Oliver, Bearden, 1983; Richins, Bloch, 1991), а в других не вызывала (Altunel, Kocak, 2017; Shaffer, Sherrell, 1997).

Хотя такие результаты могут объясняться множеством причин, из них можно выделить две главные: одну общую для корреляционного подхода как такового и одну специфическую для корреляционных исследований ПУ. Во-первых, они могут объясняться ограниченными возможностями корреляционного подхода в установлении жесткого контроля над посторонними переменными при оценке изучаемых связей. Во-вторых, они могут объясняться искажениями изучавшихся связей из-за асимметричности распределений 
измерявшихся переменных, типичной при потребительских опросах. В частности, в них часто наблюдается негативная асимметрия оценок ПУ (чрезмерная представленность высоких оценок ПУ) (Dawes et al., 2020). Такая же асимметрия неизбежно присутствует и в оценках ожиданий (поскольку потребители не склоны покупать продукты, от которых они ожидают плохого функционирования). Хотя большинство авторов названных исследований не приводили сведений о симметричности распределений оценок, некоторые из них (Oliver, Bearden, 1983; Babin et al., 1994) отмечали негативную асимметрию в полученных оценках вовлеченности. Помимо того, названные исследования обладали еще двумя ограничениями. Их авторы (кроме: Babin et al., 1994) перед расчетом связей неподтверждения с ПУ не разбивали диапазон оценок неподтверждения на области с негативным и позитивным неподтверждением, что исключало возможность раздельного изучения эффектов негативного и позитивного контраста в оценках ПУ и затрудняло точную интерпретацию полученных ими результатов (например, отсутствие предполагавшейся связи неподтверждения с ПУ могло объясняться отсутствием как обоих, так и какого-то одного из эффектов контраста). А также не изучали процессов, благодаря которым вовлеченность модерирует влияние ожиданий и функционирования на возникновение ассимиляции/контраста в оценках неподтверждения и ПУ. Другими словами, почему высокая вовлеченность усиливает воспринимаемые различия между функционированием продукта и ожиданиями, вызывая эффекты контраста?

Настоящее исследование преследовало ту же цель, что и цитировавшиеся исследования ПУ, - проверку модерирующей роли вовлеченности в возникновении ассимиляции/контраста в оценках неподтверждения и ПУ,- но отличалось от них в трех отношениях. Во-первых, проводилась более строгая проверка каузального характера связей между ожиданиями, функционированием и вовлеченностью, с одной стороны, и оценками неподтверждения и ПУ - с другой, путем проведения лабораторного эксперимента, манипулировавшего ожиданиями и функционированием продукта для создания умеренных уровней воспринимаемых различий между функционированием и ожиданиями, измерявшего неподтверждение и ПУ в ситуациях завышенных и заниженных ожиданий. Во-вторых, в нем проверялось существование обоих видов эффектов ассимиляции и контраста, позитивных и негативных. Втретьих, в нем изучались процессы, благодаря которым вовлеченность усиливает воспринимаемые различия между функционированием продукта и ожиданиями. В таком эксперименте проверялись две гипотезы. Первая касалась модерирующей роли вовлеченности в возникновении эффектов ассимиляции/контраста.

Гипотеза 1. Завышенные и заниженные ожидания к функционированию продукта будут вызывать в оченках неподтверждения ожиданий и ПУ эффекть негативного и позитивного контраста при высокой вовлеченности и эффекть позитивной и негативной ассимиляции при умеренной вовлеченности.

Вторая гипотеза касалась цепи процессов, благодаря которым вовлеченность приводит к усилению воспринимаемых различий между функционированием 
продукта и ожиданиями и последующим эффектам контраста. Теория социального суждения (Sherif, Hovland, 1961) прогнозирует, что вовлеченность субъекта сужает диапазон принятия приемлемых для него аттитюдов, из-за чего аттитюд в убеждающем сообщении оказывается в диапазоне отвержения и воспринимается более отличающимся, чем он есть в действительности (эффект контраста). Поскольку область ПУ оперирует не социальными, а перцептивными суждениями, в такое представление о диапазонах принятияотвержения следует внести три корректировки. Во-первых, как ожидания (аналог аттитюда субъекта), так и восприятия функционирования продукта (аналог аттитюда в убеждающем сообщении) формируются одним и тем же субъектом, из-за чего представления о диапазонах принятия-отвержения оказываются применимыми к обоим видам оценок. Во-вторых, под диапазонами принятия применительно к перцептивным суждениям следует понимать нижние и верхние границы оценок ожиданий и функционирования, указываемые субъектом (т.е. рассматривать такие оценки, как диапазоны оценок, варьирующих от минимальной до максимальной оценки). В-третьих, как предполагают Оливер и Бирден (Oliver, Bearden, 1983, p. 254), вовлеченность субъекта «повышает чувствительность к феноменам на выходе (т.е. к функционированию и неподтверждению)», что позволяет предположить, что она будет приводить не столько к сужению диапазона оценок ожиданий, сколько к сужению диапазона оценок восприятий функционирования продукта, из-за чего они будут становиться более точными и дифференцированными. Таким образом, с учетом этих корректировок вовлеченность должна сужать диапазон восприятий функционирования продукта, уменьшать его пересечение с диапазоном ожиданий и благодаря этому усиливать восприятие различий между функционированием и ожиданиями. Такую цепь следует дополнить еще одним звеном. Поскольку вовлеченность сопровождается усилением когнитивных процессов (Petty, Cacioppo, 1986), требующим высоких уровней активации, предполагается, что она будет повышать активацию субъекта, а уже последняя будет приводить к сужению диапазона восприятий функционирования продукта. Наконец, поскольку цепочка прогнозирует эффекты контраста как для позитивного, так и для негативного неподтверждения, вместо сырых оценок неподтверждения и удовлетворенности в ней рассматриваются абсолютные оценки, отражающие не направление, а степень неподтверждения и удовлетворенности продуктом.

Гипотеза 2. (а) Вовлеченность субъекта будет повышать уровень его активации, (б) активация будет приводить к сужению диапазона оценок воспринимаемого функционирования продукта, (в) сужение диапазона функционирования будет уменьшать его пересечение с диапазоном первоначальных ожиданий относительно продукта, (2) уменьшение такого пересечения будет усиливать степень неподтверждения ожиданий, (д) неподтверждение ожиданий будет усиливать степень удовлетворенности продуктом.

В сокращенном виде эту цепь можно описать следующим образом: вовлеченность $\rightarrow$ активация $\rightarrow$ диапазон восприятий функционирования $\rightarrow$ пересечение диапазонов восприятий функционирования и ожиданий $\rightarrow$ степень неподтверждения $\rightarrow$ степень удовлетворенности. 


\section{Метод}

Для исследования на основе метода снежного кома набирались 120 участников (47\% мужчин) от 18 до 59 лет (средний возраст - 26 лет), которые при помощи блоковой рандомизации распределялись в одно из восьми условий факторного плана 2 (сравнительно низкое/высокое качество спрея) $\times 2$ (обычные/специфические ожидания к качеству спрея) $\times 2$ (умеренная/высокая вовлеченность). Цель исследования озвучивалась как оценка различных марок спреев для очистки экранов мониторов ${ }^{1}$.

Стимульный материал. В качестве продукта использовался спрей Techpoint. В ходе пилотажного тестирования с 10 дополнительными участниками на его основе путем разбавления жидким мылом или водой изготавливались несколько образцов, из которых для дальнейшего исследования были отобраны два, вызывавшие средние оценки 7 и 4 балла по шкале от 0 (полное отсутствие пятен/разводов) до 10 (очень много пятен/разводов) и соответствующие умеренно низкому и умеренно высокому уровню качества спрея.

Процедура. Исследование проводилось индивидуально. Прибывшему участнику зачитывалась инструкция: В этом исследовании оценивается способность средств, предназначенных для очистки экранов мониторов, к удалению одного из самых сильных загрязнений, следов жирных пальщев. Сегодня вам предстоит оценить два средства из числа тех, которые мы отобрали для исследования. После этого участнику эксперимента предлагали заполнить анкету (пол, возраст, частота использования спреев для экранов мониторов, вовлеченность и ожидания к качеству оцениваемого спрея). Во время тренировки участнику показывали, а затем его просили трижды повторить процедуру удаления воображаемого пятна с экрана монитора, контролируя степень прижатия губки, обернутой салфеткой с нанесенным на нее спреем, количество и широту круговых движений. Затем предлагали удалить с экрана следы двух пятен (для удаления второго пятна ему выдавалась новая салфетка с нанесенным на нее спреем), которые наносились на его глазах в центральной области экрана на расстоянии примерно 15 см друг от друга путем прижатия большого пальца сначала к сливочному маслу, а затем к экрану. Далее участника просили подождать 1 минуту для получения окончательных результатов работы спрея, предлагали заполнить анкету, измерявшую уровень их активации, восприятие качества спрея, неподтверждение ожиданий, удовлетворенность и покупательские намерения, с ним проводили дебрифинг, благодарили и отпускали.

Экспериментальные манипулящии. Исследование манипулировало тремя независимыми переменными: качество спрея, ожидания и вовлеченность.

Манипулящии качеством спрея. В условиях со сравнительно низким качеством спрея участникам предлагали оценить целевой (т.е. изучавшийся) образец, вызывавший в пилотажном тестировании среднюю оценку 7 баллов, а в условиях со сравнительно высоким качеством 4 балла.

\footnotetext{
${ }^{1}$ Эксперимент проводился С. Нерсисян и Д. Садыковой.
} 
Манипулящии ожиданиями к качеству спрея. В условиях с обычными ожиданиями участники сразу оценивали целевой образец либо сравнительно низкого (7 баллов), либо сравнительно высокого качества (4 балла), тогда как в условиях со специфическими ожиданиями перед целевым образцом участникам предлагали оценить образец противоположного уровня качества (4 и 7 баллов соответственно). Для них такое задание выглядело как последовательная оценка двух марок спреев. В отличие от условий с обычными ожиданиями это позволяло сформировать у них перед оценкой целевого образца завышенные или заниженные ожидания (по аналогии с манипулированием праймами в исследованиях социальных восприятий - Stapel, Suls, 2007) с последующим негативным или позитивным неподтверждением ожиданий. Промежуток времени между тестированием спреев в условиях со специфическим ожиданиями составлял 3-4 минуты.

Манипулящии вовлеченностью. В исследовании изучались эффекты ситуационной вовлеченности из-за ее большей подверженности маркетинговым воздействиям в сравнении с устойчивой вовлеченностью (Bloch, Richins, 1983; Houston, Rothschild, 1978). Поскольку она требует манипулирования важностью для субъекта последствий рассматриваемой проблемы (Petty, Cacioppo, 1986; Petty, Briñol, 2012), в условиях высокой вовлеченности зачитывавшаяся участникам инструкция дополнялась словами: Ваши оценки будут иметь два важных последствия. Во-первых, мь доведем результать этого исследования до будущих покупателей, что облегчит их выбор при покупке подобных чистящих средств. Во-вторых, поскольку некоторые из них были разработань российскими компаниями, мы доведем эти результаты до них, что поможет им успешнее конкурировать со средствами, которые производят зарубежнье компании.

Зависимые переменные. Манипуляции ожиданиями и восприятием качества спрея измерялись 10-сантиметровой графической шкалой с границами, обозначающими «полное отсутствие пятен/разводов» и «очень много пятен/разводов». В обоих случаях участников сначала просили проставить на ней свои оценки крестиком, а затем при помощи двух вертикальных линий указать минимальные и максимальные оценки, которые они дали бы такому спрею. Манипуляции вовлеченностью измерялись шкалой семантического дифференциала (Mittal, 1995) с просьбой оценить задание на оценку чистящих средств как неважное - важное, не имеет - имеет ко мне отношение, не заботит - заботит меня, не затрагивает - затрагивает меня лично, не имеет имеет для меня значение (альфа Кронбаха = 0.88). Уровень активации участников измерялся 10-пунктовой подшкалой активности из анкеты САН (Еникеев, 2003) (альфа Кронбаха = 0.85). Неподтверждение ожиданий измерялось просьбой оценить результаты применения спрея по шкале от 1 (гораздо больше пятен/разводов) до 7 (гораздо меньше пятен/разводов). Удовлетворенность качеством спрея измерялась шкалой от -4 (чрезвычайно не удовлетворен) до 4 (чрезвычайно удовлетворен), а вероятность его покупки шкалой от -4 (чрезвычайно маловероятно) до 4 (чрезвычайно вероятно). Поскольку две последние оценки сильно коррелировали, они были объединены 
в общую оценку удовлетворенности (альфа Кронбаха = 0.93). В условиях со специфическими ожиданиями оценки ожиданий, восприятий качества, неподтверждения ожиданий, удовлетворенности и вероятности покупки собирались только для второго (целевого) образца спрея. В дальнейшем для облегчения интерпретации оценки ожиданий и восприятий качества спрея переводились в обратные.

\section{Результаты}

Проверка манипуляций. Для проверки успешности манипуляций независимыми переменными реализовывались три ANCOVA 2 (сравнительно низкое/высокое качество спрея) $\times 2$ (обычные/специфические ожидания) $\times 2$ (умеренная/высокая вовлеченность). Поскольку из трех контролируемых переменных - пола, возраста и частоты использования спрея - последняя имела позитивные связи с восприятиями качества спрея, неподтверждением и удовлетворенностью $(r=0.31,0.35$ и 0.35$)$, она включалась во все эти и последующие ANCOVA в роли ковариата. (Такие связи свидетельствуют о большей чувствительности к воспринимаемым различиям между функционированием спрея и ожиданиями у более опытных пользователей спреев для очистки экранов мониторов.) Скорректированные с учетом ковариата средние оценки и стандартные ошибки восприятий качества спрея, ожиданий и вовлеченности в разных экспериментальных условиях показаны в таблице 1.

Таблица 1

Средние и стандартные ошибки (в скобках) восприятий качества спрея, ожиданий и вовлеченности в разных экспериментальных условиях

\begin{tabular}{|c|c|c|c|c|c|c|c|}
\hline \multicolumn{4}{|c|}{ Сравнительно низкое качество спрея } & \multicolumn{4}{|c|}{ Сравнительно высокое качество спрея } \\
\hline \multicolumn{2}{|c|}{$\begin{array}{c}\text { Умеренная } \\
\text { вовлеченность }\end{array}$} & \multicolumn{2}{|c|}{$\begin{array}{c}\text { Высокая } \\
\text { вовлеченность }\end{array}$} & \multicolumn{2}{|c|}{$\begin{array}{c}\text { Умеренная } \\
\text { вовлеченность }\end{array}$} & \multicolumn{2}{|c|}{$\begin{array}{c}\text { Высокая } \\
\text { вовлеченность }\end{array}$} \\
\hline $\begin{array}{c}\text { Обычные } \\
\text { ожидания }\end{array}$ & $\begin{array}{c}\text { Завышен- } \\
\text { ные ожи- } \\
\text { дания }\end{array}$ & $\begin{array}{c}\text { Обычные } \\
\text { ожида- } \\
\text { ния }\end{array}$ & $\begin{array}{c}\text { Завышен- } \\
\text { ные ожи- } \\
\text { дания }\end{array}$ & $\begin{array}{c}\text { Обычные } \\
\text { ожида- } \\
\text { ния }\end{array}$ & $\begin{array}{c}\text { Завышен- } \\
\text { ные ожида- } \\
\text { ния }\end{array}$ & $\begin{array}{c}\text { Обычные } \\
\text { ожида- } \\
\text { ния }\end{array}$ & $\begin{array}{c}\text { Завышен- } \\
\text { ные ожи- } \\
\text { дания }\end{array}$ \\
\hline $\mathrm{M}(\mathrm{SE})$ & $\mathrm{M}(\mathrm{SE})$ & $\mathrm{M}(\mathrm{SE})$ & $\mathrm{M}(\mathrm{SE})$ & $\mathrm{M}(\mathrm{SE})$ & $\mathrm{M}(\mathrm{SE})$ & $\mathrm{M}(\mathrm{SE})$ & $\mathrm{M}(\mathrm{SE})$ \\
\hline \multicolumn{8}{|c|}{ Восприятия качества спрея } \\
\hline $3.76(0.49)$ & $4.60(0.48)$ & $3.37(0.48)$ & $2.93(0.49)$ & $8.15(0.48)$ & $6.01(0.48)$ & $8.23(0.51)$ & $5.15(0.48)$ \\
\hline \multicolumn{8}{|c|}{ Ожидания к спрею } \\
\hline $5.11(0.45)$ & $6.63(0.44)$ & $6.44(0.44)$ & $7.32(0.44)$ & $6.68(0.44)$ & $4.59(0.44)$ & $6.38(0.47)$ & $5.49(0.44)$ \\
\hline \multicolumn{8}{|c|}{ Вовлеченность } \\
\hline$-0.42(0.21)$ & $0.03(0.21)$ & $0.69(0.21)$ & $0.82(0.21)$ & $0.06(0.21)$ & $-0.28(0.21)$ & $0.94(0.23)$ & $0.87(0.21)$ \\
\hline
\end{tabular}

Примечание. $\mathrm{n}=15$ в каждой ячейке. Увеличение средних отражает увеличение оценок соответствующих переменных. 
Как и ожидалось, анализ оценок восприятий качества спрея обнаружил основной эффект качества, $F(1,111)=81.34, p<0.001$, partial $\omega^{2}=0.40$, и ожиданий, $F(1,111)=12.33, p<0.001$, partial $\omega^{2}=0.09$, квалифицировавшихся эффектом взаимодействия качества и ожиданий, $F(1,111)=16.67, p<0.001$, partial $\omega^{2}=0.12$. Участники выше оценивали спрей со сравнительно высоким качеством, чем со сравнительно низким ( $M=6.89$ vs. 3.66), при этом такие различия сильнее проявлялись при обычных ожиданиях. В дополнение также обнаружился основной эффект вовлеченности, $F(1,111)=4.20, p<0.05$, partial $\omega^{2}=0.03:$ участники выше оценивали качество спрея при умеренной, чем при высокой вовлеченности ( $M=5.63$ vs. 4.92). Остальные эффекты были незначимыми. Как и ожидалось, анализ оценок ожиданий обнаружил эффект взаимодействия качества и ожиданий, $F(1,111)=18.31, p<0.001$, partial $\omega^{2}=0.13$ : в условиях завышенных ожиданий участники формировали более высокие ожидания к спрею, чем в условиях обычных ожиданий ( $M=6.97$ vs. 5.77$)$, а в условиях заниженных ожиданий, наоборот, более низкие ( $M=5.03$ vs. 6.53). В дополнение также обнаружился основной эффект вовлеченности, $F(1,111)=4.34$, $p<0.05$, partial $\omega^{2}=0.03$ : участники демонстрировали более высокие ожидания при высокой, чем при умеренной вовлеченности ( $M=6.41$ vs. 5.75). Остальные эффекты были незначимыми. Наконец, как и ожидалось, анализ оценок вовлеченности обнаружил основной эффект вовлеченности, $F(1,111)=41.71$, $p<0.001$, partial $\omega^{2}=0.25$ : участники демонстрировали более высокие оценки вовлеченности при высокой, чем при умеренной вовлеченности $(M=0.83$ vs. -0.15$)$. Остальные эффекты были незначимыми. Таким образом, манипуляции независимыми переменными были успешными. В дополнение к этому также следует отметить, что оценки восприятий качества спрея были близки к оценкам пилотажного исследования и что в оценках ожиданий, восприятий качества, вовлеченности, неподтверждения и удовлетворенности не наблюдалось заметной асимметрии (ее значения варьировались от -0.28 до 0.13), а сами оценки охватывали теоретически возможные диапазоны.

Проверка первой гипотезы. Для проверки первой гипотезы реализовывались ANCOVA оценок неподтверждения ожиданий и удовлетворенности спреем. Как и предполагалось, анализ оценок неподтверждения ожиданий обнаружил трехсторонний эффект взаимодействия, $F(1,111)=13.08, p<0.001$, partial $\omega^{2}=0.09$, свидетельствующий о том, что эффекты взаимодействия ожиданий и вовлеченности различались при сравнительно низком и высоком качестве спрея. Для более детального изучения этот эффект раздроблялся на двухсторонние эффекты взаимодействия ожиданий и вовлеченности отдельно для спрея со сравнительно низким и высоким качеством. Оба двухсторонних эффекта оказались значимыми, $F(1,111)=6.53$ и 6.33 , обе $p<0.05$, оба partial $\omega^{2}=0.04$. Как и прогнозировалось в первой гипотезе, завышенные ожидания к функционированию спрея вызывали эффект негативного контраста при высокой вовлеченности и эффект позитивной ассимиляции при умеренной. В левой части рисунка 1 видно, что при высокой вовлеченности завышенные ожидания к спрею сравнительно низкого качества приводили к более негативным 
оценкам неподтверждения в сравнении с обычными ожиданиями, тогда как при умеренной, наоборот, к менее негативным.

Как и прогнозировалось в первой гипотезе, заниженные ожидания к функционированию спрея вызывали эффект позитивного контраста при высокой вовлеченности и эффект негативной ассимиляции при умеренной. В правой части рисунка 1 показано, что при высокой вовлеченности заниженные ожидания к спрею сравнительно высокого качества приводили к более позитивным оценкам неподтверждения в сравнении с обычными ожиданиями, тогда как при умеренной, наоборот, к менее позитивным.

В дополнение к этому также обнаружился эффект частоты использования спрея, $F(1,111)=6.45, p<0.05$, partial $\omega^{2}=0.04$, и основной эффект качества, $F(1,111)=133.54, p<0.001$, partial $\omega^{2}=0.52$, квалифицировавшийся эффектом взаимодействия качества и вовлеченности, $F(1,111)=4.53, p<0.05$, partial $\omega^{2}=0.03$. Участники испытывали негативное неподтверждение ожиданий при оценке спрея со сравнительно низким качеством и позитивное при оценке спрея с высоким качеством ( $M=2.42$ vs. 5.23), при этом такие различия сильнее проявлялись при высокой вовлеченности ( $M=2.07$ vs. 5.38$)$, чем при умеренной $(M=2.77$ vs. 5.08). Все остальные эффекты были незначимыми.

Как и в предыдущем анализе, ANCOVA оценок удовлетворенности обнаружил трехсторонний эффект взаимодействия, $F(1,111)=10.86, p<0.001$, partial $\omega^{2}=0.08$, который для более детального изучения раздроблялся на двухсторонние эффекты взаимодействия ожиданий и вовлеченности отдельно для спрея со сравнительно низким и высоким качеством. Однако в отличие от предыдущего анализа значимым оказался первый, $F(1,111)=11.12, p<0.01$, partial $\omega^{2}=0.08$, но не второй эффект, $F(1,111)=1.67, p>0.05$, partial $\omega^{2}=0.01$. Как и прогнозировалось в первой гипотезе, завышенные ожидания к функционированию спрея вызывали эффект негативного контраста при высокой

Рисунок 1

Средние оценки неподтверждения ожиданий в разных экспериментальных условиях
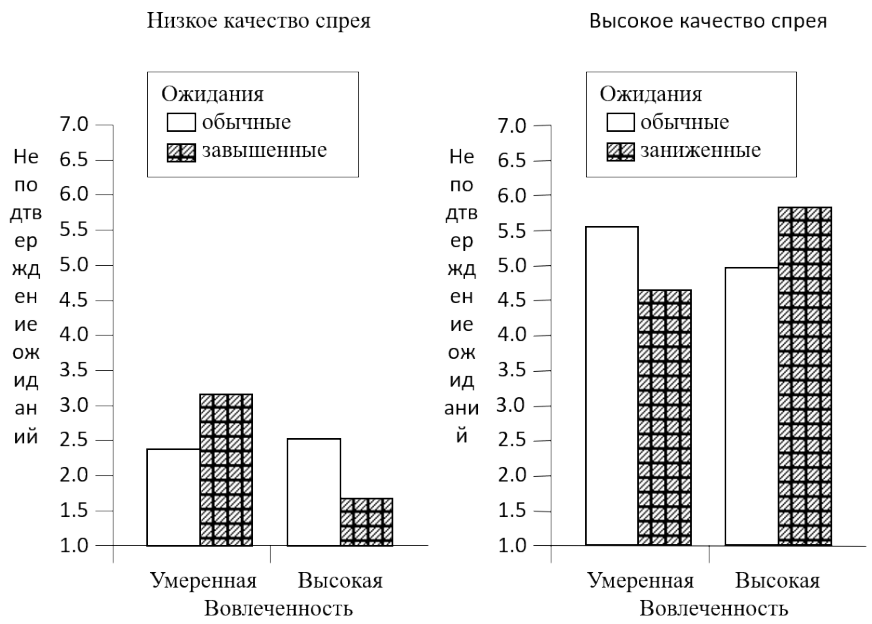
Рисунок 2

Средние оценки удовлетворенности в разных экспериментальных условиях
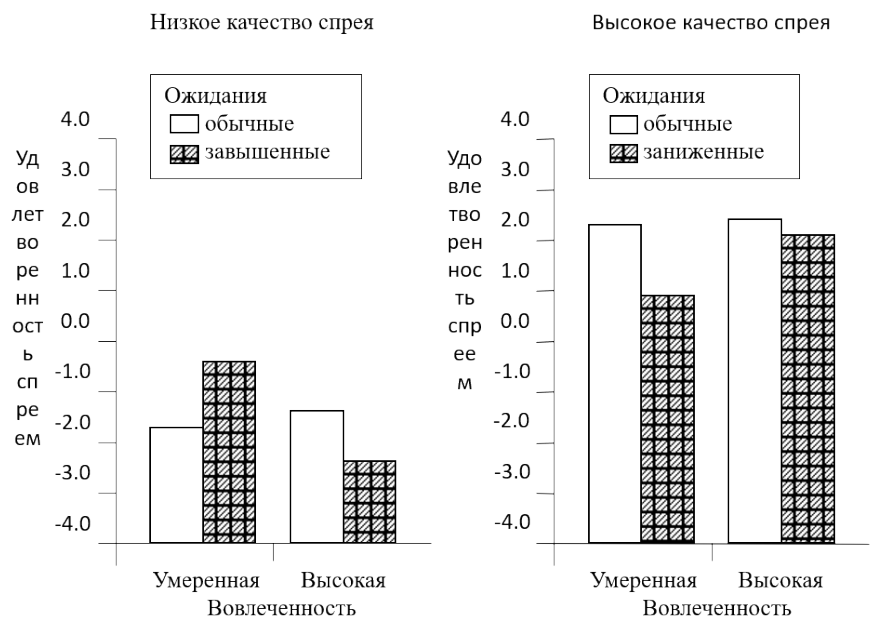

вовлеченности и эффект позитивной ассимиляции при умеренной. В левой части рисунка 2 видно, что при высокой вовлеченности завышенные ожидания к спрею сравнительно низкого качества приводили к более негативным оценкам удовлетворенности, тогда как при умеренной, наоборот, к менее негативным.

Однако вопреки прогнозам первой гипотезы, хотя заниженные ожидания к функционированию спрея и вызывали эффект негативной ассимиляции при умеренной вовлеченности, они не вызывали эффекта позитивного контраста при высокой вовлеченности. В правой части рисунка 2 показано, что если при умеренной вовлеченности заниженные ожидания к спрею сравнительно высокого качества действительно приводили к менее позитивным оценкам удовлетворенности, то при высокой вовлеченности они не приводили к более позитивным оценкам. В последнем случае не наблюдалось ни позитивного контраста, ни негативной ассимиляции.

В дополнение к этому, как и в предыдущем анализе оценок неподтверждения ожиданий, обнаружился основной эффект качества, $F(1,111)=111.91, p<0.001$, partial $\omega^{2}=0.48$, квалифицировавшийся эффектом взаимодействия качества и вовлеченности, $F(1,111)=4.09, p<0.05$, partial $\omega^{2}=0.03$. Все остальные эффекты были незначимыми.

Таким образом, результаты ANCOVA свидетельствуют в пользу первой гипотезы для неподтверждения и частично для удовлетворенности.

Проверка второй гипотезы. Для проверки гипотезы в отношении процессов, благодаря которым вовлеченность приводит к усилению воспринимаемых различий между функционированием и ожиданиями и последующим эффектам контраста - вовлеченность $\rightarrow$ активация $\rightarrow$ диапазон восприятий функционирования $\rightarrow$ пересечение диапазонов восприятий функционирования 
и ожиданий $\rightarrow$ степень неподтверждения $\rightarrow$ степень удовлетворенности,реализовывался путевой анализ (path analysis). Оценки диапазона восприятий функционирования ${ }^{2}$ были следствием нахождения разностей между максимальными и минимальными оценками восприятий качества спрея; оценки пересечения диапазонов восприятий функционирования и ожиданий получались путем ранжирования разностей между максимальными оценками восприятий качества и минимальными оценками ожиданий для спреев с низким качеством и между максимальными оценками ожиданий и минимальными оценками восприятий качества для спреев с высоким качеством; оценки степени неподтверждения и удовлетворенности рассчитывались нахождением абсолютных значений оценок неподтверждения и удовлетворенности после вычитания из тех и других средних выборочных оценок. Итоговая путевая модель приведена на рисунке 3.

Во-первых, активация регрессировалась на вовлеченность. Как и ожидалось, связь между ними была позитивной $(\beta=0.32)$. Во-вторых, оценки диапазона восприятий качества регрессировались на обе предыдущие переменные. Как и ожидалось, они обладали негативной связью только с активацией $(\beta=-0.21)$. В-третьих, оценки пересечения диапазонов восприятий функционирования и ожиданий регрессировались на три предыдущие переменные. Как и ожидалось, они обладали позитивной связью только с диапазоном вос-

\section{Влияние вовлеченности на воспринимаемые различия между функционированием} и ожиданиями, неподтверждение и удовлетворенность

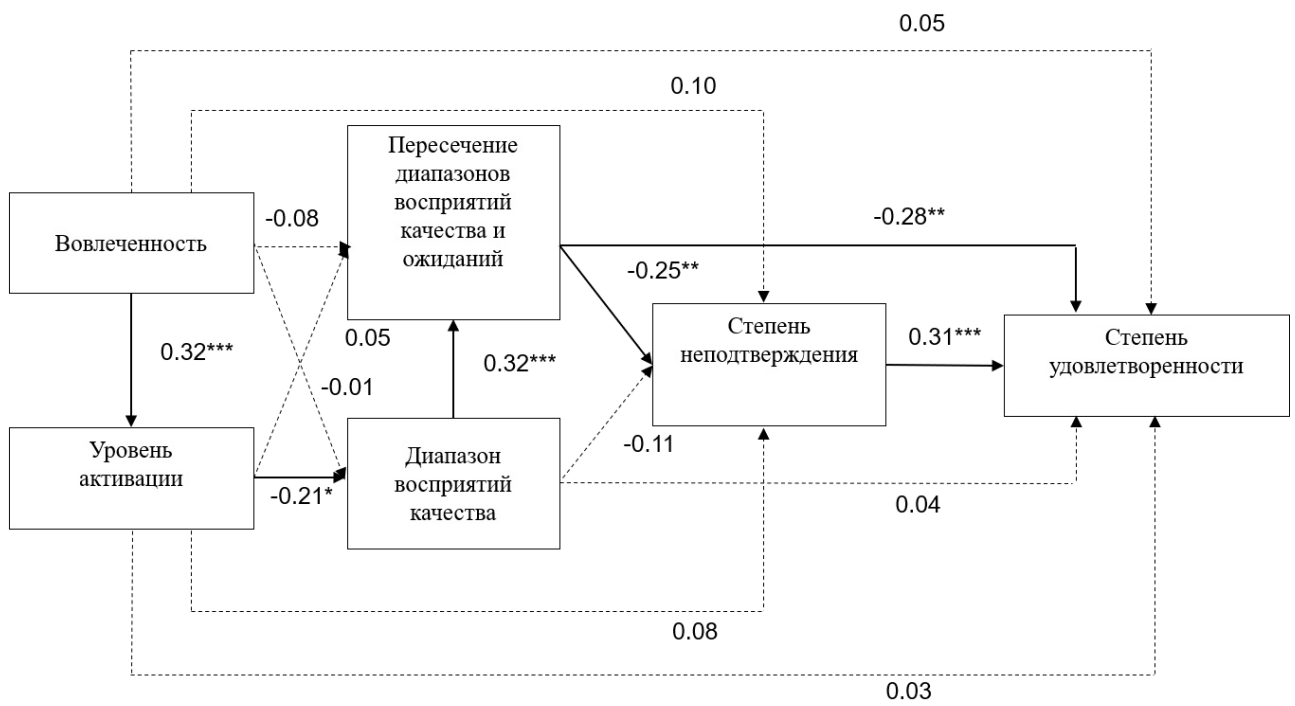

Примечание. Приведены стандартизированные $\beta$-коэффициенты. Сплошные стрелки отражают значимые пути, а пунктирные - незначимые пути. ${ }^{*} p<0.05$. ${ }^{* *} p<0.01 .{ }^{* * *} p<0.001$.

\footnotetext{
${ }^{2}$ В рассматриваемом эксперименте под функционированием понимается качество спрея.
} 
приятий качества $(\beta=0.32)$. В-четвертых, оценки степени неподтверждения регрессировались на все предыдущие переменные. Как и ожидалось, они обладали негативной связью только с пересечением диапазонов восприятий функционирования и ожиданий $(\beta=-0.25)$. Наконец, оценки степени удовлетворенности регрессировались на все предыдущие переменные. Как и ожидалось, они обладали позитивной связью со степенью неподтверждения $(\beta=0.31)$. В дополнение они также имели негативную связь с пересечением диапазонов восприятий функционирования и ожиданий $(\beta=-0.28)$, указывающую, что такое пересечение может играть относительно независимую роль в формировании удовлетворенности, это согласуется с ролью диапазонов принятияотвержения в возникновении эффектов контраста в теории социального суждения (Sherif, Hovland, 1961). В дополнение к оценке связей между переменными в цепи процессов на рисунке 3 также проверялась возможность того, что каждая промежуточная переменная опосредовала связь между предшествующей и следующей за ней переменной. Тесты на опосредование Соубела (Baron, Kenny, 1986) показали, что это происходило во всех случаях (все $z$ варьировались от -2.17 до -1.87 , все $p<0.05)$, кроме одного: степень неподтверждения не опосредовала связи диапазона восприятий с удовлетворенностью $(z=-1.11, p>0.05)$. Таким образом, путевой анализ поддержал вторую гипотезу в отношении процессов, благодаря которым вовлеченность усиливает воспринимаемые различия между функционированием и ожиданиями, вызывающие эффекты контраста.

Результаты исследования в целом поддержали гипотезу о модерирующей роли вовлеченности в эффектах ассимиляции/контраста и согласуются с результатами единственного предыдущего эксперимента (Babin et al., 1994), проверявшего сходную гипотезу, но не манипулировавшего ожиданиями и функционированием продукта с целью создания различных степеней неподтверждения ожиданий, а просто его измерявшего с разбиением участников на группы с разными уровнями неподтверждения. А также гипотезу о цепи процессов, благодаря которым вовлеченность выполняет свою модерирующую роль в возникновении эффектов контраста, акцентирующую роль когнитивных процессов в восприятии различий между функционированием и ожиданиями и вытекающую из теории социального суждения (Sherif, Hovland, 1961) и модели вероятности осмысления (Petty, Cacioppo, 1986).

Вместе с тем в отличие от второй первая гипотеза не получила полной поддержки, поскольку в исследовании не обнаружился один из четырех эффектов, прогнозировавшихся в оценках удовлетворенности,- эффект позитивного контраста. Существуют несколько объяснений такого результата. Первое связано с недостаточной эффективностью манипуляций заниженными ожиданиями: ожидания у высокововлеченных участников оказывались недостаточно низкими для возникновения позитивного контраста. Второе состоит в том, что таким участникам в принципе присущи более высокие ожидания, что должно приводить к бо́льшим воспринимаемым различиям между функционированием и ожиданиями при завышенных, чем при заниженных ожиданиях. Такое объяснение вытекает из проверки манипуляций ожиданиями в 
этом исследовании, обнаружившей основной эффект вовлеченности. Такой феномен уже наблюдался ранее (Bolfing, Woodruff, 1988; Shaffer, Sherrell, 1997) и может объясняться большей заинтересованностью высокововлеченных субъектов в хорошем функционировании продукта. Наконец, третье объяснение состоит в том, что манипуляции важными последствиями оказались смешанными с манипуляциями самопрезентацией (Johnson, Eagly, 1989). Последнее могло побуждать участников сообщать менее поляризованные оценки удовлетворенности, т.е. более низкие при завышенных ожиданиях и более высокие при заниженных. Это могло не влиять на сообщение оценок неподтверждения, поскольку они могли думать, что до производителей и других потребителей будут доводиться только оценки качества и/или удовлетворенности спреем. Для проверки того, какое из этих трех объяснений адекватно, потребуются другие исследования.

Настоящее исследование имеет ограничения: проверка второй гипотезы опиралась на путевой анализ, т.е. по сути на корреляции, ограничивающие возможность каузальных интерпретаций связей, в особенности в последних звеньях процессов, приводящих к эффектам контраста; изучение единичного продукта, что обусловливает необходимость в его репликации на других продуктах; измерение ряда переменных однопунктовыми шкалами, что могло снижать надежность оценок. Однако, с другой стороны, поскольку снижение надежности приводит к ослаблению наблюдаемых эффектов связей или различий (Nunnally, Bernstein, 1994), при большей надежности оценок результаты проверки гипотез могли бы оказаться еще более выраженными.

Результаты исследования поднимают ряд вопросов. Например, при проверке манипуляций качеством обнаружился основной эффект вовлеченности, снижавшей оценки восприятий качества спрея. Исчезал бы такой эффект, если бы в оценках удовлетворенности наблюдался не только негативный, но и позитивный контраст (восприятия качества и удовлетворенность коррелировали в этом исследовании, $r=0.76$ ), или он является естественным следствием более высоких ожиданий вовлеченных субъектов? Не обнаружилось связей диапазона ожиданий, в отличие от диапазона восприятий, с другими переменными. Но что произошло бы, если бы участникам предлагали самим выбирать для тестирования марки спреев и/или сообщали их характеристики? Не привело бы это к сужению не только диапазона восприятия, но и диапазона ожиданий у высокововлеченных участников и к еще большему усилению эффектов контраста? Косвенно на такую возможность указывают исследования, обнаружившие влияние на неподтверждение ожиданий уверенности в ожиданиях (Spreng, Page, 2001; Yi, La, 2003). В будущих исследованиях можно было бы также проверить альтернативные объяснения эффектов ассимиляции/ контраста, исходящие из области социальных восприятий. Например, модель избирательной доступности (Mussweiler, 2003, 2007) и установки/сброса (Martin et al., 1990; Martin, Shirk, 2007). Первая объясняет их формулированием субъектом предварительной гипотезы о сходстве или различии между объектом и используемым стандартом оценки, а вторая - его низким или высоким вниманием к своим подлинным реакциям на объект, побуждающим 
либо не корректировать (установка), либо корректировать возможную предвзятость при его оценке (сброс).

Из эффектов ассимиляции/контраста и модерирующей роли вовлеченности могут вытекать практические следствия для рынка. Поскольку удовлетворенность продуктом влияет на повторные покупки и прибыли компании, представляется важным разрабатывать новые продукты, которые бы обеспечивали высокую удовлетворенность у целевых потребителей. Для чего необходимо, во-первых, изучать ожидания потребителей к существующим продуктам, а во-вторых, добиваться чтобы характеристики новых продуктов сильно превосходили такие ожидания, так как лишь это обеспечит сильное позитивное неподтверждение ожиданий и последующие эффекты позитивного контраста. Но поскольку обычно бывает трудно достичь сильного превосходства нового продукта, может оказаться достаточным добиться его умеренного превосходства, если повысить вовлеченность потребителей в процесс покупательского выбора, что может стимулировать интерес к изучению ситуационных факторов вовлеченности, которые бы могли контролироваться производителями и продавцами. При этом в силу универсальности эффектов ассимиляции/контраста их можно использовать с целью повышения удовлетворенности в различных сферах жизни. Например, те же принципы (оценка ожиданий, формирование предложения, умеренно превышающего ожидания, и усиление вовлеченности) могут оказаться полезными в области государственного управления (удовлетворенность граждан), организационного управления (удовлетворенность работников), семейного консультирования (удовлетворенность супругов) и др.

\section{Выводы}

Результаты исследования поддержали модерирующую роль вовлеченности при возникновении эффектов ассимиляции/контраста, а также гипотетическую цепь процессов, в которой вовлеченность повышает уровень активации субъекта, активация стимулирует его когнитивную активность, когнитивная активность увеличивает точность суждений в оценках продукта (сужая диапазон восприятий функционирования продукта), а точность суждений усиливает воспринимаемые различия между функционированием и ожиданиями (уменьшая его пересечение с диапазоном ожиданий), что увеличивает степень неподтверждения и удовлетворенности. Вместе с тем в отличие от неподтверждения ожиданий в исследовании не был обнаружен эффект позитивного контраста в оценках удовлетворенности. Предлагаются объяснения отсутствия позитивного, но не негативного контраста в оценках удовлетворенности.

\section{Литература}

Еникеев, М. И. (2003). Психологическая диагностика. Стандартизированные тесты. М.: ПРИОР.

Ссылки на зарубежные источники см. в разделе References после англоязычного блока. 
Ребзуев Борис Геннадьевич - доцент, Институт психологии, Федеральное государственное бюджетное образовательное учреждение высшего образования «Российский государственный педагогический университет им. А.И. Герцена», кандидат психологических наук, доцент.

Сфера научных интересов: организационное поведение, поведение потребителя.

Контакты: rebzuevbg@herzen.spb.ru

\title{
The Role of Involvement in the Occurrence of Assimilation/Contrast in Consumer Satisfaction Scores
}

\author{
B.G. Rebzuev ${ }^{\mathrm{a}}$ \\ ${ }^{a}$ The Herzen State Pedagogical University of Russia, 48 Moika Emb., St. Petersburg, 191186, Russian \\ Federation
}

\begin{abstract}
The research shows that one of the key determinants of consumer satisfaction is disconfirmation, which refers to the perceived differences between product performance and pre-purchase expectations. In this case, positive disconfirmation (when performance exceeds expectations) leads to high, and negative (when it concedes to expectations) to low satisfaction. The present study examined the role of involvement in the occurrence of assimilation effects (a decrease in disconfirmation scores and a shift in satisfaction scores towards expectations) and contrast effects (an increase in disconfirmation scores and a shift in satisfaction scores in the opposite direction), as well as the processes leading to contrast effects. And two hypotheses have been formulated that involvement will produce contrast effects and that such effects will result from the activation of cognitive processes leading to increased perceived differences between product performance and expectations. The hypotheses were tested in a laboratory experiment that manipulated product performance (a screen cleaner quality), expectations and participant involvement levels, and explored two types of assimilation/contrast effects, negative and positive, resulting from high and low expectations for the product. The experiment showed that involvement did produce negative and positive contrast effects in the disconfirmation and satisfaction scores with one exception: involvement did not produce positive contrast effect in the satisfaction scores. The article discusses the possible reasons for this result. The experiment also supported the existence of a chain of processes leading to contrast effects, in which involvement increases the activation levels in subjects, activation stimulates cognitive activity, cognitive activity increases judgment accuracy in product evaluations, and judgment accuracy enhances perceived differences between product performance and expectations. Limitations, questions for future research and implications for manufacturers and retailers from assimilation/contrast effects are discussed.
\end{abstract}

Keywords: involvement, consumer satisfaction, expectancy disconfirmation, expectations, product performance, assimilation effect, contrast effect, latitude of acceptance, latitude of rejection, social judgment theory, elaboration likelihood model. 


\section{References}

Altunel, M. C., \& Kocak, O. E. (2017). The roles of subjective vitality, involvement, experience quality, and satisfaction in tourists' behavioral intentions. European Journal of Tourism Research, 16, 233251. https://ejtr.vumk.eu/index.php/about/article/view/287

Babin, B. J., Griffin, M., \& Babin, L. (1994). The effect of motivation to process on consumers' satisfaction reactions. In C. T. Allen \& D. R. John (Eds.), Advances in consumer research: Vol. 21 (pp. 406-411). Provo, UT: Association for Consumer Research.

Baron, R. M., \& Kenny, D. A. (1986). The moderator-mediator variable distinction in social psychological research: Conceptual, strategic, and statistical considerations. Journal of Personality and Social Psychology, 51(6), 1173-1182. https://doi.org/10.1037//0022-3514.51.6.1173

Bloch, P. H., \& Richins, M. L. (1983). A theoretical model for the study of product importance perceptions. Journal of Marketing, 47(3), 69-81. https://doi.org/10.2307/1251198

Bolfing, C. P., \& Woodruff, R. B. (1988). Effects of situational involvement on consumers' use of standards in satisfaction/dissatisfaction processes. Journal of Consumer Satisfaction/dissatisfaction and Complaint Behavior, 1, 16-24.

Curtis, T., Abratt, R., Rhoades, D. L., \& Dion, P. (2011). Customer loyalty, repurchase and satisfaction: A meta-analytical review. Journal of Consumer Satisfaction, Dissatisfaction and Complaining Behavior, 24, 1-26.

Dawes, J., Stocchi, L., \& Dall'Olmo-Riley, F. (2020). Over-time variation in individual's customer satisfaction scores. International Journal of Market Research, 62(3), 262-271. https://doi.org/10.1177/1470785320907538

Enikeev, M. I. (2003). Psikhologicheskaya diagnostika. Standartizirovannye testy [Psychological diagnostics. Standardized tests]. Moscow: PRIOR.

Houston, M. L., \& Rothschild, M. L. (1978). Conceptual and methodological perspectives on involvement. In S. C. Jain (Ed.), Educator's proceedings (pp. 184-187). Chicago, IL: American Marketing Association.

Johnson, B. T., \& Eagly, A. H. (1989). Effects of involvement on persuasion: A meta-analysis. Psychological Bulletin, 106(2), 290-314. https://doi.org/10.1037/0033-2909.106.2.290

Martin, L. L., Seta, J. J., \& Crelia, R. A. (1990). Assimilation and contrast as a function of people's willingness and ability to expend effort in forming an impression. Journal of Personality and Social Psychology, 59(1), 27-37. https://doi.org/10.1037/0022-3514.59.1.27

Martin, L. L., \& Shirk, S. (2007). Set/reset and self-regulation: Do contrast processes play a role in the breakdown of self-control? In D. A. Stapel \& J. Suls (Eds.), Assimilation and contrast in social psychology (pp. 207-225). Psychology Press.

Mittal, B. (1995). A comparative analysis of four scales of consumer involvement. Psychology $\mathcal{E}$ Marketing, 12(7), 663-682. https://doi.org/10.1002/mar.4220120708

Mussweiler, T. (2003). Comparison processes in social judgment: Mechanisms and consequences. Psychological Review, 110(3), 472-489. https://doi.org/10.1037/0033-295X.110.3.472

Mussweiler, T. (2007). Assimilation and contrast as comparison effects: A selective accessibility model. In D. A. Stapel \& J. Suls (Eds.), Assimilation and contrast in social psychology (pp. 165-185). Psychology Press.

Nunnally, J. C., \& Bernstein, I. (1994). Psychometric theory (3rd ed.). New York, NY: McGraw-Hill.

Oliver, R. L. (2014). Satisfaction: A behavioral perspective on the consumer: A behavioral perspective on the consumer (2nd ed.). New York, NY: Routledge. 
Oliver, R. L., \& Bearden, W. O. (1983). The role of involvement in satisfaction processes. In R. P. Bagozzi \& A. M. Tybout (Eds.), Advances in consumer research: Vol. 10 (pp. 250-255). Provo, UT: Association for Consumer Research.

Olshavsky, R. W., \& Miller, J. A. (1972). Consumer expectations, product performance, and perceived product quality. Journal of Marketing Research, 9(1), 19-21. https://doi.org/10.2307/3149600

Petty, R. E., \& Briñol, P. (2012). The elaboration likelihood model. In P. A. M. Van Lange, A. Kruglanski, \& E. T. Higgins (Eds.), Handbook of theories of social psychology (pp. 224-245). London, England: Sage Publications Ltd. https://doi.org/10.4135/9781446249215.n12

Petty, R. E., \& Cacioppo, J. T. (1986). Communication and persuasion: Central and peripheral routes to attitude change. New York, NY: Springer-Verlag. https://doi.org/10.1007/978-1-4612-4964-1

Richins, M. L., \& Bloch, P. H. (1991). Post-purchase product satisfaction: Incorporating the effects of involvement and time. Journal of Business Research, 23(2), 145-158. https://doi.org/10.1016/0148-2963(91)90025-S

Shaffer, T. R., \& Sherrell, D. L. (1997). Consumer satisfaction with healthcare services: The influence of involvement. Psychology \& Marketing, 14(3), 261-285. https://doi.org/10.1002/(SICI)15206793(199705)14:3<261::AID-MAR4>3.0.CO;2-9

Sherif, M., \& Hovland, C. I. (1961). Social judgment: Assimilation and contrast effects in communication and attitude change. Oxford: Yale University Press.

Spreng, R. A., \& Page, T. (2001). The impact of confidence in expectations on consumer satisfaction. Psychology \& Marketing, 18(11), 1187-1204. https://doi.org/10.1002/MAR.1049

Spreng, R. A., \& Sonmez, E. (2000). The moderating effect of involvement on the consumer satisfaction formation process. In American Marketing Association Proceeding (Vol. 11, pp. 168-174). Chicago, IL: American Marketing Association.

Stapel, D. A., \& Suls, J. (2007). (Eds.). Assimilation and contrast in social psychology. New York, NY: Psychology Press. https://doi.org/10.4324/9780203837832

Szymanski, D. M., \& Henard, D. H. (2001). Customer satisfaction: A meta-analysis of the empirical evidence. Journal of the Academy of Marketing Science, 29(1), 16-35. https://doi.org/10.1177/0092070301291002

Tam, J. L. M. (2011). The moderating effects of purchase importance in customer satisfaction process: An empirical investigation. Journal of Consumer Behaviour, 10(4), 205-215. https://doi.org/10.1002/cb.330

Woodruff, R. B., Cadotte, E. R., \& Jenkins, R. L. (1983). Modeling consumer satisfaction processes using experience-based norms. Journal of Marketing Research, 20(3), 296-304. https://doi.org/10.2307/3151833

Yi, Y. (1990). A critical review of consumer satisfaction. In V. A. Zeithaml (Ed.), Review of marketing (pp. 68-123). Chicago, IL: American Marketing Association.

Yi, Y., \& La, S. (2003). The moderating role of confidence in expectations and the asymmetric influence of disconfirmation on customer satisfaction. The Service Industries Journal, 23(5), 20-47. https://doi.org/10.1080/02642060308565622

Zimbardo, P. G. (1960). Involvement and communication discrepancy as determinants of opinion conformity. Journal of Abnormal and Social Psychology, 60(1), 86-94. https://doi.org/10.1037/h0040786

Boris G. Rebzuev - Associate Professor, Institute of Psychology, The Herzen State Pedagogical University of Russia, PhD in Psychology, Associate Professor.

Research Area: Organizational behavior, consumer behavior.

E-mail: rebzuevbg@herzen.spb.ru 